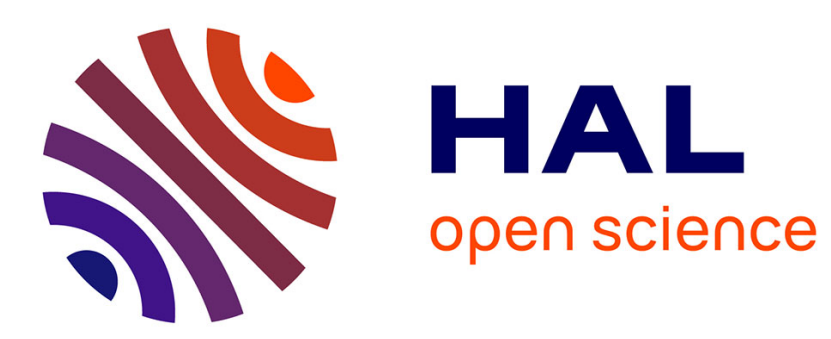

\title{
An Objective Evaluation of Edge Detection Methods Based on Oriented Half Kernels
}

Baptiste Magnier

\section{To cite this version:}

Baptiste Magnier. An Objective Evaluation of Edge Detection Methods Based on Oriented Half Kernels. Alamin Mansouri, Abderrahim El Moataz, Fathallah Nouboud, Driss Mammass. Image and Signal Processing, 10884, Springer, pp.80-89, 2018, Lecture Notes in Computer Science, 978-3-31994210-0. 10.1007/978-3-319-94211-7_10. hal-01940247

\section{HAL Id: hal-01940247 https://hal.science/hal-01940247}

Submitted on 30 Nov 2018

HAL is a multi-disciplinary open access archive for the deposit and dissemination of scientific research documents, whether they are published or not. The documents may come from teaching and research institutions in France or abroad, or from public or private research centers.
L'archive ouverte pluridisciplinaire HAL, est destinée au dépôt et à la diffusion de documents scientifiques de niveau recherche, publiés ou non, émanant des établissements d'enseignement et de recherche français ou étrangers, des laboratoires publics ou privés. 


\title{
An Objective Evaluation of Edge Detection Methods based on Oriented Half Kernels
}

\author{
Baptiste Magnier \\ IMT Mines d'Alès, LGI2P, 6. avenue de Clavières 30100 Alès, France \\ Baptiste.Magnier@mines-ales.fr
}

\begin{abstract}
Easy to use, oriented half kernels are reliable in image analysis. These thin filters, rotated in all the desired directions are useful to detect edges, or extract precisely their orientations, even concerning highly noisy images. Usually, the filtering process corresponds to convolutions with Gaussians and their derivatives. Other filters exist and can be implemented in order to build half kernels. However, functions used for the smoothing and derivative parts have not been studied in depth. The goal of this paper is to evaluate different types of half filters as a function of the noise level. The studied kernels have the same spatial support, enabling easier comparisons. To address the robustness of the studied filters against noise, the image quality is gradually worsened. Then, their performances are compared through objective evaluations of both segmentation and gradient direction.
\end{abstract}

Keywords: Edge detection, half kernels, gradient direction, evaluation.

\section{Introduction}

Edge detection is a fundamental process and remains widely used in image analysis and computer vision applications. Moreover, gradient and edge orientations are essential information for the interpretation and exploitation of digital images 26/19. Classical methods use first-order fixed operators 21/5|24|4] or the structure tensor [1] to detect gradient and its orientation. Gradient-based edge and orientation estimators are frequently utilized. Among these operators, DoB (Difference of Boxes) filters 21] often remain implemented in many applications as in robotics. DoB simply computes the difference between two mean filters of same sizes on both sides of the considered pixel (usually represented by a list of 1 or -1). However, DoB filters are sensitive to noise and orientations not reliable. The robustness of the detection is generally improved by smoothing the

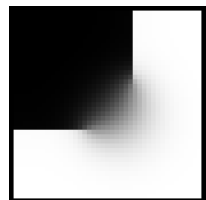

(a)Blurred corner (b) DoB [21]

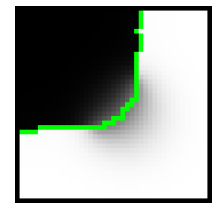

(c) Gaussian [5]

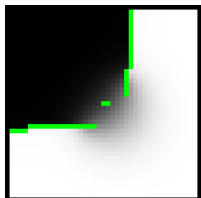

(d) AGK 9

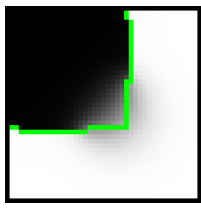

(e) Half kernel

Fig. 1. Edge extraction around a blurred corner using different filters. 


\begin{tabular}{|l|c|c|c|}
\hline Type of operator & Fixed operator & Oriented Filters & Half Kernels \\
\hline Example of filter & & & \\
\hline Gradient magnitude & $|\nabla I|=\sqrt{I_{0}^{2}+I_{\pi / 2}^{2}}$ & $|\nabla I|=\max _{\theta \in[0, \pi[}\left|I_{\theta}\right|$ & $|\nabla I|=\max _{\theta \in[0,2 \pi[} I_{\theta}-\min _{\theta \in[0,2 \pi[} I_{\theta}$ \\
\hline Gradient direction & $\eta=\arctan \left(\frac{I_{\pi / 2}}{I_{0}}\right)$ & $\eta=\underset{\theta \in[0, \pi[}{\arg \max }\left|I_{\theta}\right|+\frac{\pi}{2}$ & $\eta=\left(\underset{\theta \in[0,2 \pi[}{\arg \max } I_{\theta}+\underset{\theta \in[0,2 \pi[}{\arg \min } I_{\theta}\right) / 2$ \\
\hline
\end{tabular}

Table 1. Gradient magnitude and orientation computation for a scalar image $I$ where $I_{\theta}$ represents the image derivative at the $\theta$ orientation (in radians).

image by a low pass filter followed by a gradient detection using vertical and horizontal masks of type $\left[\begin{array}{lll}-1 & 0 & 1\end{array}\right]$. Edge detection methods differ in the types of smoothing filters that are applied [5|24]6/4, as equations presented in Tab 3 . The computations of the gradient magnitude $|\nabla I|$ and its orientation $\eta$ are presented in Tab 11(left). Among all the edge detectors, box [21] and exponential [24|4] filters do not delocalize contour points [12, whereas they are sensitive to noise and blur while contours near a corner are rounded by Gaussian and Deriche filters (cf. Fig. 2(a)). In the orientation and edge detection domains, steerable feature detectors represent popular and efficient tools $[8|10| 9$. This concept provides valuable orientations and edges for many image processing applications, especially when the filter is elongated (cf. Fig. 2(b)). The concept was generalized in 20] by decomposing a given filter kernel optimally in a set of basic filters approximating an Anisotropic Gaussian Kernel (AGK). Mathematically, Tab11(middle column) presents the orientation and the gradient magnitude computation using this type of filter, corresponding to the maximum energy in the maximal response direction. The AGK possesses a common shortcoming, as a matter of fact, only one $\pi$-periodic orientation is extracted efficiently [20, so the impossibility of these filters to estimate in a relevant way several coexisting orientations at the same pixel, as illustrated in Fig. 11(d). Otherwise, multi steerable filters are designed and adjusted to a pattern of interest or a region to determine more precisely the directions of edges with $2 \pi$-periodic templates 23|18|13. On the other hand, the asymmetric filter developed in [16] estimates the homogeneity in multiple local directions. This filter consists in a network of several parallel lines in which a homogeneity is computed and enables an estimation of edge directions modulo $2 \pi$. Lastly, wedge 23[18 or asymmetric oriented filters [15]16] sound better suited to multiple edge directions detection or modeling a template. The next section details the edge detection process using $2 \pi$-periodic filters.

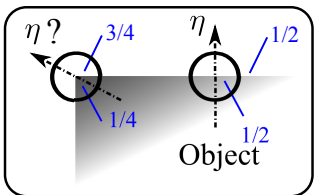

(a) Circular support

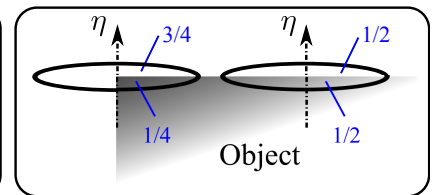

(b) Elongated support (AGK)

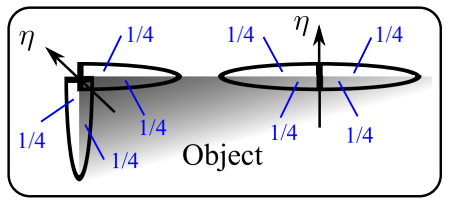

(c) Half kernel (HK) support

Fig. 2. Representation of filter supports concerning edges and corners. 


\section{Edge detection using Half Oriented Kernels}

\subsection{Advantages of half oriented kernels}

Edge detection techniques using elongated kernels are efficient to detect large linear structures correctly [10/20/922]. The robustness against noise depends strongly on the smoothing parameters of the filter, i.e., the parameter of the filter elongation. If the filter length increases, the detection is less sensitive to noise, but small structures are considered as noise and their edges are not extracted. Consequently, the accuracy of the detected edge points decreases strongly at corner points and for non-straight object contour parts, as illustrated in Fig. 1(d). To bypass this undesirable effect, an anisotropic edge detection method is developed in [17] and [15]. Indeed, the proposed technique is able to detect crossing edges and corners due to two elongated and oriented filters in two different directions. The simplest solution is to consider paths crossing each pixel in several directions. The idea proposed in [17] and [15] is to "cut" the elongated kernel using a Heaviside function into two parts, i.e. two directions (see Fig. 2(c)). Then, the half kernel (HK) is rotated in several directions from 0 to $2 \pi$ (bilinear rotation) and computes a derivative information at each desired angle (illustrated in Fig. 3. for each $\pi / 36$ radian angle). Thus, in a contour direction, a half smoothing is performed, whereas in the perpendicular direction, a derivative information is computed, as illustrated in Fig. 3(a),(d)-(g). In order to better understand this technique to extract edge, the filter support of a HK on a straight contour is equivalent to $1 / 2$ on both sides of the edge, as for elongated and circular or box filters, illustrated in Fig. 2 . On the contrary, for a corner point with a $\pi / 2$ radian angle, the support of the half filter remains $1 / 2$ on both sides of the edge, whereas it is around $1 / 4$ and $3 / 4$ concerning other filter supports. Such a manner to extract edges enables to compute a gradient of blurred contours, even corners
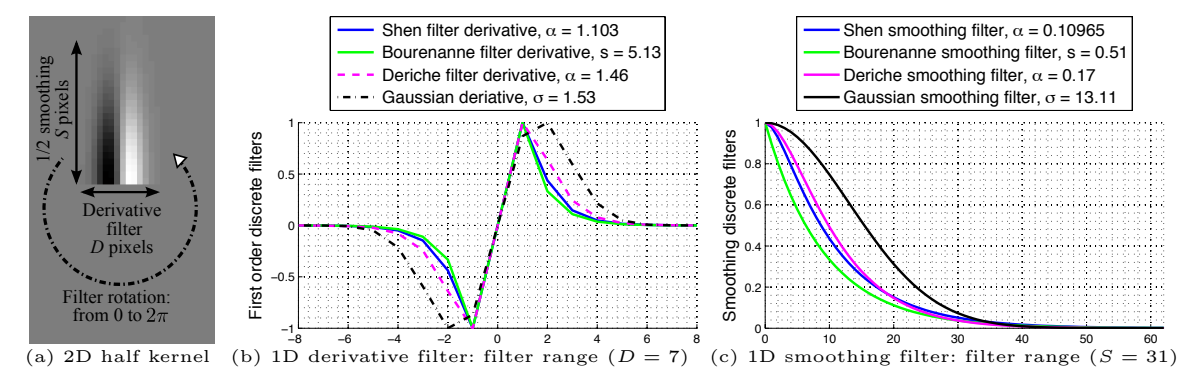

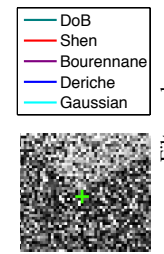

(d) Legend

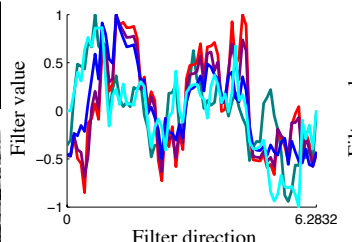

(e) $D=5, S=11$

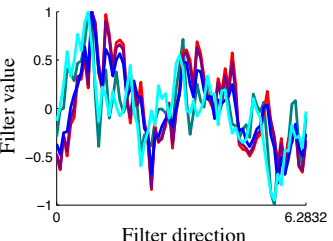

(f) $D=5, S=21$

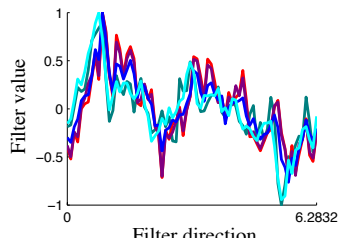

Filter direction
$D=7, S=31$

Fig. 3. Decomposition of half kernel (HK) in one dimension. Normalized signals in (e)-(g) represent the filter response (counter-clockwise rotation) of the centered pixel in (d), with $D$ and $S$ the derivative and smoothing parameters (cf. Tab 4 and 5 ). 


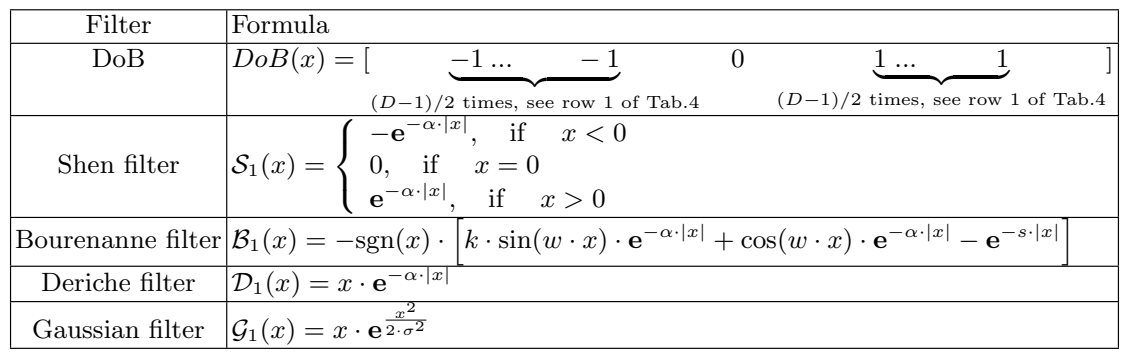

Table 2. Different derivative filters. Parameters are available in Tab 3

as illustrated in Fig. 11(e), contrary to other filtering methods. Eventually, as detailed in Tab.1(right), the gradient corresponds to the maximum value minus the minimum value of the convolution of the oriented half filter with the image. These two directions represent to the two main orientations of a contour and the gradient direction $\eta$ corresponds to the bisector between these two directions (cf. Fig. 2(c)). These orientations are useful and efficient for image restoration via PDE 14], corner detection [3] or image descriptor [25].

\subsection{Types of implemented filters}

Edge detection using oriented half kernels is reliable, robust against noise, and, outperforms classical edge detectors [1514]. As pointed in [17] and illustrated in Figs 5(1)-(q), the HK can be implemented using different equations:

- a derivative part of the filter, represented by an equation in Tab 2

- a half smoothing part, which corresponds to an equation in Tab3.

In order to create an elongated filter, the support of the half smoothing part must be higher than the derivative support. The parameters of the different filter equations can be chosen in Tab.5 for the half smoothing part, where $S$ represents the length of the support where $98 \%$ of the filtered information is computed, i.e., the width of the HK (cf. Fig $3(\mathrm{c})$ ). As for the derivative part, the parameters are available in Tab 4 , where $D$ represents the length of the support where $98 \%$ of the filtered information is computed (i.e., width of the HK, cf. Fig 3 (b)). As an example, a half filter using Bourennane equation, of length $S=46$ pixels and $D=11$ pixels, the parameter corresponds to $s=3.22$ for the smoothing part and $s=0.33$ for the derivative part. The half filter can be generated using a

\begin{tabular}{|c|c|c|}
\hline Filter & Formula & Parameter(s) \\
\hline Boxes & $\operatorname{Box}(x)=[$ & $S \in \mathbb{N}^{*}$ \\
\hline & $S$ times, see row 1 of $\mathrm{Tab} 5$ & \\
\hline Shen filter & $\mathcal{S}(x)=\mathbf{e}^{-\alpha \cdot|x|}$ & $\alpha \in \mathbb{R}_{+}^{*}$ \\
\hline Bourenanne filter & $\begin{array}{l}\mathcal{B}(x)=\frac{\mathbf{e}^{-\alpha \cdot|x|}}{\alpha^{2}+w^{2}} \cdot[-(k \cdot \alpha+w) \cdot \sin (w \cdot|x|)+ \\
\left.(\alpha-k \cdot w) \cdot \cos (w \cdot x) \cdot \mathbf{e}^{-\alpha \cdot|x|}\right]-\frac{\mathbf{e}^{-s \cdot|x|}}{s}\end{array}$ & $\begin{array}{l}s \in \mathbb{R}_{+}^{*}, w=0.01 \\
k=-0.564 \\
\alpha=0.215 \cdot s\end{array}$ \\
\hline Deriche filter & $\mathcal{D}(x)=(\alpha \cdot|x|+1) \cdot \mathbf{e}^{-\alpha \cdot|x|}$ & $\alpha \in \mathbb{R}_{+}^{*}$ \\
\hline Gaussian filter & $\mathcal{G}(x)=\mathbf{e}^{\frac{x^{2}}{2 \cdot \sigma^{2}}}$ & $\sigma \in \mathbb{R}_{+}^{*}$ \\
\hline
\end{tabular}

Table 3. Different low pass filters compared in this study. 
half DoB, see first rows in Tab2 and Tab. Thus, $S$ and $D$ numbers, indicate directly the size of the half DoB, which is rotated from 0 to $2 \pi$. It is important to note that the parameters of the half smoothing parts in Tab 5 correspond to parameters representing a pass bas filter centered at 0 , and only the causal part is preserved here, so $S$ corresponds to a smoothing support size of a half filter. As a result, the derivative part computes the oriented gradient information, whereas the half smoothing part enables a smooth along edges, preserving them and their directions. Finally, on the one hand, the length of the HK must be sufficiently large to be robust against noise. On the other hand, the width of the HK must be thin to ensure precise edge detection and orientations. As an example in Fig. 3(left), when $S$ and $D$ are small, all the signals are noisy, whereas when $S$ increases, the edge directions highlight incrementally, even though Shen and Bourennane HK remain always sensitive to noise.

The Matlab code is available on the MathWorks website: https://fr.mathnorks.com/ matlabcentral/fileexchange/66853-edge-detection-methods-based-on-oriented-half-kernels?s_tid=srchtitle.

\section{Evaluation and Results}

The aim of the experiments is to obtain the best edge map in a supervised way. In the experiments, 7 edge detection methods based on filtering gradient computation are compared: isotropic Gaussian filter [5], AGK 9 and HK formed by the DoB, Shen [24], Bourennane [4, Deriche [6], and Gaussian filters [1715]. These filters possess the same spatial support (AGK has two times the spacial support of the HK, Fig $5(\mathrm{~m}))$. Filters must be thin and elongated in order to compute accurate contours and associated directions (cf. Fig. 3(d)-(g)). Thus, the derivative parameter concerning isotropic Gaussian filter [5] remains the same than others $(D=7)$, see Fig $5(\mathrm{l})-(\mathrm{q})$. HK and AGK are rotated each $\pi / 72$ radian angles. Finally, after a non-maximum suppression [26], an objective assessment is performed by varying hysteresis thresholds on normalized thin edges until the Relative Distance Error (RDE) 7] evaluation obtains the minimum score [2]:

\begin{tabular}{|c|c|c|c|c|c|c|c|c|c|}
\hline Spacial support: Number of pixels $D$ & 3 & 5 & 7 & 9 & 11 & 13 & 15 & 17 & 19 \\
\hline Shen filter: values of $\alpha$ & 2.15 & 1.52 & 1.10 & 0.86 & 0.69 & 0.58 & 0.49 & 0.43 & 0.38 \\
\hline Bourenanne filter: values of $s$ & 9.99 & 7.07 & 5.13 & 3.98 & 3.22 & 2.69 & 2.3 & 2 & 1.77 \\
\hline Deriche filter: values of $\alpha$ & 3.16 & 2.01 & 1.46 & 1.14 & 0.93 & 0.79 & 0.68 & 0.6 & 0.49 \\
\hline Gaussian filter: values of $\sigma$ & 0.7 & 1.11 & 1.53 & 1.95 & 2.38 & 2.8 & 3.23 & 3.66 & 4.09 \\
\hline
\end{tabular}

Table 4. Parameters of the filters in function of their support size, i.e., the number of pixels under the range of the filter. These parameters correspond to the derivative part. The first row is tied to the range of the derivative of the DoB filter.

\begin{tabular}{|c|c|c|c|c|c|c|c|c|c|c|c|}
\hline Spacial support $S$ & 16 & 21 & 26 & 31 & 36 & 41 & 46 & 51 & 56 & 61 & 66 \\
\hline Shen filter: $\alpha$ & 0.23 & 0.17 & 0.133 & 0.11 & 0.095 & 0.082 & 0.071 & 0.065 & 0.058 & 0.052 & 0.047 \\
\hline Bourenanne filter: $s$ & 1.05 & 0.78 & 0.62 & 0.51 & 0.44 & 0.38 & 0.33 & 0.3 & 0.27 & 0.24 & 0.22 \\
\hline Deriche filter: $\alpha$ & 0.33 & 0.25 & 0.2 & 0.17 & 0.14 & 0.12 & 0.11 & 0.1 & 0.09 & 0.08 & 0.07 \\
\hline Gaussian filter: $\sigma$ & 6.66 & 8.81 & 10.96 & 13.11 & 15.26 & 17.41 & 19.56 & 21.7 & 23.85 & 26 & 28.15 \\
\hline
\end{tabular}

Table 5. Parameters of the half filters in function of their support size. These parameters are used for the length of the $2 \mathrm{D}$ filters, i.e., smoothing part. The first row is tied to the range of the mean filter used in the DoB filter, i.e., number of pixels $S$. 


$$
\operatorname{RDE}\left(G_{t}, D_{c}\right)=\sqrt{\frac{1}{\left|D_{c}\right|} \cdot \sum_{p \in D_{c}} d_{G_{t}}^{2}(p)}+\sqrt{\frac{1}{\left|G_{t}\right|} \cdot \sum_{p \in G_{t}} d_{D_{c}}^{2}(p)},
$$

where $D_{c}$ represents the desired contour map, $G_{t}$ the ground truth edge image (Fig. $5(\mathrm{~b})),|\cdot|$ the number of contour points of a set, and, $d_{A}(p)$ is the minimal Euclidian distance between a pixel $p$ and a set $A$ [71]. These scores are recorded and plotted as a function of the noise level in the original image, as presented in Fig 5(j). Hence, a plotted curve increases monotonously with the noise level (Gaussian noise). The second evaluation measure concerns the gradient angle, $\eta$ (cf. Tab 1). Once $D_{c}$ is created, considering $\mathcal{C}_{D_{c}}$ the set of contour chains in $D_{c}$ (i.e., at least 2 pixels per chain), the gradient evaluation as follows:

$$
E\left(\mathcal{C}_{D_{c}}, \eta\right)=\frac{1}{\left|\mathcal{C}_{D_{c}}\right|} \cdot \sum_{p \in \mathcal{C}_{D_{c}}} \sum_{d_{k} \in W}\left[1-\frac{a b s\left(\pi / 2-a b s\left(\overrightarrow{\eta_{p}}-\overrightarrow{\eta_{d_{k}}}\right)\right)}{\pi / 2}\right] / c_{k},
$$

where $d_{k}$ represents a contour pixel belonging to $W$, a $3 \times 3$ window centered on $p, \overrightarrow{\eta_{d_{k}}}$ the gradient orientation of $d_{k}$ and $c_{k}$ the number of contour pixels in $W$, minus the central pixel. This evaluation linearly ranges from 0 for identical angles of $\overrightarrow{\eta_{p}}$ and $\overrightarrow{\eta_{d_{k}}}$ to 1 for angles that differs (Fig 4 . Note that angles of $\overrightarrow{\eta_{d_{k}}}$ and $\overrightarrow{\eta_{p}}$ belong to $[0 ; \pi[$ and when one direction approximates 0 and the other direction $\pi$, the evaluation of these two directions remains close to 0 (see Fig 4(c)).

The presented segmentations in Fig 5 correspond to the original image for a PSNR $=14 \mathrm{~dB}$. Clearly, all the HK obtains better segmentations than the isotropic Gaussian. The HK using Gaussian outperforms six other compared filters, in terms of both RDE evaluation and visualization. AGK obtains good scores, but the contour image presents undesirable straight contours disturbing its interpretation. HK using DoB produces many false positive points, especially close to the true edges; it performs in presence of salt\&pepper noise, but not concerning white Gaussian noise. Otherwise, HK using Shen and Bourennane filters obtain close the same results, however, they remain too sensitive to noise, and, especially, many edges are missing. HK with Deriche filter is more robust to noise but edges are still missing. Concerning $\eta$ evaluation, $E\left(\mathcal{C}_{D_{c}}, \eta\right)$ indicates that HK using Gaussian remains more reliable than other HK. This information means that HK using Gaussian is less disturbed by noise than other kernels. The gradient evaluation of AGK is close to 0 because almost all the edges obtained are straight; consequently, the gradient angle remains close the same for all the contour chain.

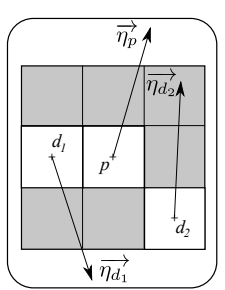

(a) $\overrightarrow{\eta_{p}}$ and $\overrightarrow{\eta_{d_{k}}}$

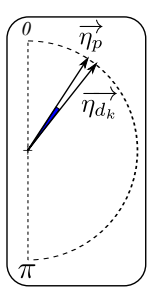

(b) $e \approx 0$

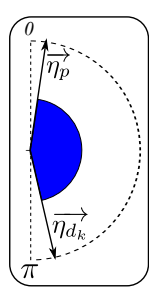

(c) $e \approx 0.1$

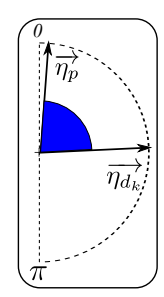

(d) $e \approx 0.9$

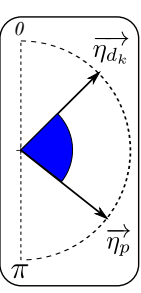

(e) $e \approx 1$

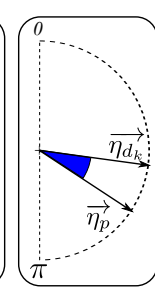

(f) $e \approx 0.3$

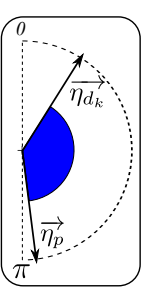

(g) $e \approx 0.5$

Fig. 4. Illustration of $\eta_{p}$ and $\eta_{d_{k}}$ directions, in a $3 \times 3$ window, where each white square represents a contour pixel of $D_{c}$, whereas gray squares correspond to non-edge pixels. Example of angular single error computation $e=1-a b s\left(\pi / 2-a b s\left(\eta_{p}-\eta_{d_{k}}\right)\right) / \pi / 2$. 


\section{Conclusion}

Oriented half kernels (HK) represent thin filters enable to describe the image information all around a considered pixel. As they are robust against noise and their edge directions are accurate, HK are utilized in the context of many image processing problems 14/3/25. This study presents different manners to build HK devoted to edge detection in digital images. Five HK have been objectively compared and HK using Gaussian remains the most reliable. Especifically, this HK and the anisotropic Gaussian kernel obtain similar evaluation results kernel but the obtained edges are more usable with HK. For this purpose, we plan in a future study to implement multiscale and subpixel half Gaussian kernels.

\section{References}

1. Abdulrahman, H. Magnier, B. and Montesinos, P.: From contours to ground truth: How to evaluate edge detectors by filtering. In: J. of WSCG, 25(2), 133-142, (2017)

2. Abdulrahman, H. Magnier, B. and Montesinos, P.: A new objective supervised edge detection assessment using hysteresis thresholds. In International Workshop on Brain-Inspired Computer Vision, held as part of ICIAP, 3-14, (2017)

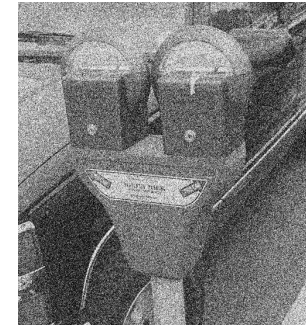

(a) $\mathrm{PSNR}=14 \mathrm{~dB}$

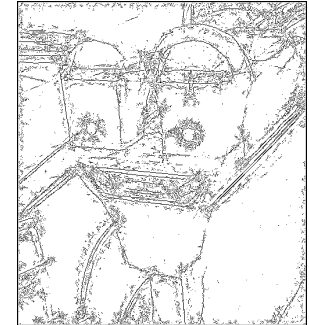

(e) HK: DoB

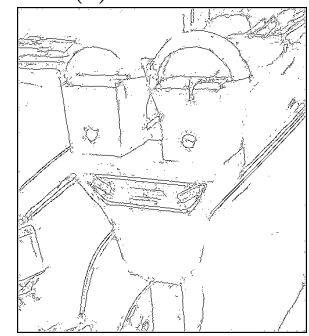

(i) HK: Gaussian

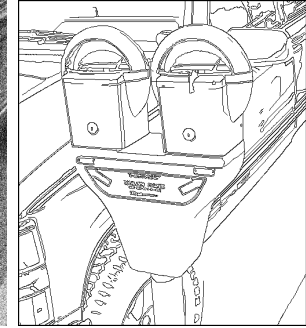

(b) $G_{t}$ image 1

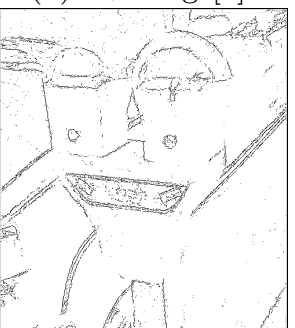

(f) HK: Shen

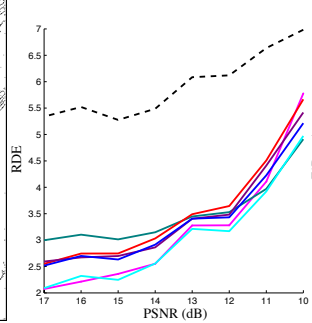

(j) RDE

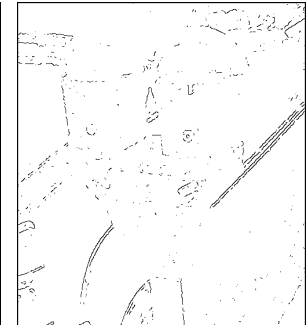

(c) Gaussian $[5$

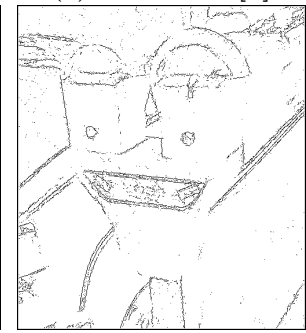

(g) HK: Bourennane

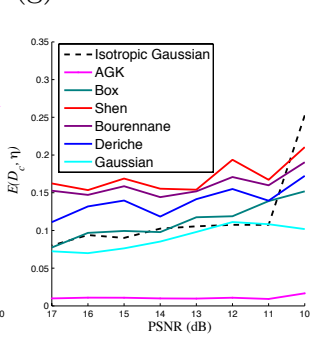

(k) $E\left(\mathcal{C}_{D_{c}}, \eta\right)$

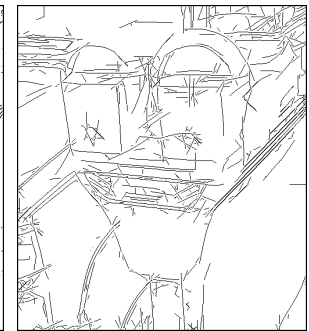

(d) AGK 9

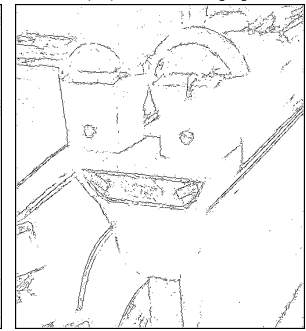

(h) HK: Deriche

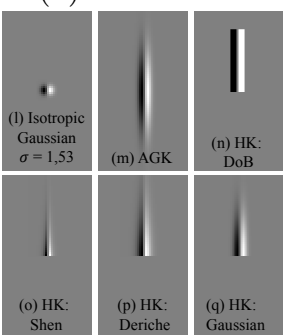

HK, $D=7$ and $S=31$

Fig. 5. Edge detection corresponding of the minimum score of the RDE measure. 
3. Abdulrahman, H., Magnier, B., and Montesinos, P.: Oriented asymmetric kernels for corner detection. In: IEEE EUSIPCO, pp. 778-782, (2017)

4. Bourennane, E. Gouton, P. Paindavoine, M. and Truchetet, F.: Generalization of Canny-Deriche filter for detection of noisy exponential edge. In Sign. Proc., 82(10), 1317-1328, (2002)

5. Canny, J. F.: A computational approach to edge detection. In: IEEE TPAMI. 8(6), 679-698, (1986)

6. Deriche, R.: Using Canny's criteria to derive a recursively implemented optimal edge detector. In: Int. J. Computer Vision, 1, 167-187, (1987)

7. Dubuisson, M.P. and Jain, A.K.: A modified Hausdorff distance for object matching, In: 12th IAPR Int. Conf. on Pattern Recognition: 1, 566-568, (1994)

8. Freeman, W. T. and Adelson, E. H.: The design and use of steerable filters. In: IEEE TPAMI: 13, 89-906, (1991)

9. Geusebroek, J.M., Smeulders, A. and van de Weijer, J.: Fast anisotropic gauss filtering. In: ECCV, 99-112, (2002)

10. Jacob, M. and Unser, M.: Design of steerable filters for feature detection using Canny-like criteria. In: IEEE TPAMI, 26(8), 1007-1019, (2004)

11. Köthe, U.: Reliable low-level image analysis. In: Habilitation thesis, (2007)

12. Laligant, O. Truchetet, F. and Meriaudeau, F.:Regularization preserving localization of close edges. In: IEEE Signal Proc. Letters, 14(3), 185-188, (2007)

13. Magnier, B., Montesinos, P., and Diep, D.: Texture removal by pixel classification using a rotating filter. In: IEEE ICASSP, pp. 1097-1100, (2011)

14. Magnier, B. and Montesinos, P.: Evolution of image regularization with PDEs toward a new anisotropic smoothing based on half kernels. In IS\&T/SPIE Electronic Imaging, International Society for Optics and Photonics, 86550M-86550M, (2013)

15. Magnier, B., Montesinos, P. and Diep, D.: Fast anisotropic edge detection using gamma correction in color images. In: IEEE ISPA, 212-217, (2011)

16. Michelet, F., Da Costa, J.-P., Lavialle, O., Berthoumieu, Y., Baylou, P. and Germain, C.: Estimating local multiple orientations. In: Sign. Proc., 87(7), pp. 16551669, (2007)

17. Montesinos, P. and Magnier, B.: A new perceptual edge detector in color images. In: ACIVS, Springer, Berlin, Heidelberg, pp. 209-220, (2010)

18. Mühlich, M., Friedrich, D., and Aach, T. Design and implementation of multisteerable matched filters. In: IEEE TPAMI, 34(2), 279-291, (2012)

19. Papari, G. and Petkov, N.: Edge and line oriented contour detection: State of the art. In: Image and Vision Computing: 29(2) , 79-103, (2011)

20. Perona, P.: Steerable-scalable kernels for edge detection and junction analysis. In: IVC, $10(10)$, pp. 663-672, (1992)

21. J. M. S. Prewitt: Object enhancement and extraction. In: Picture Processing and Psychopictorics, B. Lipkin and A. Rosenfeld. Eds. Academic Press, New York, (1970)

22. Püspöki, Z., Martin, S., Sage, D. and Unser, M.: Transforms and operators for directional bioimage analysis: a survey. In: Focus on Bio-Image Informatics, Springer, pp. 69-93, (2016)

23. Simoncelli, E. P. and Farid, H.: Steerable wedge filters for local orientation analysis. In: IEEE TIP, 5(9), pp. 1377-1382, Citeseer, (1996)

24. Shen, J. and Castan, S.:An optimal linear operator for edge detection. In: IEEE CVPR, 86, 109-114, (1986)

25. Venkatrayappa, D., Montesinos, P., Diep, D., and Magnier, B.: A Novel Image Descriptor Based on Anisotropic Filtering. In CAIP, Springer, pp. 161-173, (2015)

26. Ziou, D. and Tabbone, S.: Edge detection techniques: an overview. In: Int. J. on Pat. Rec. and Image Anal., 8(4), 537-559, (1998) 